\title{
On the Modeling of the Production and Drift of Carriers in Cyclohexane
}

\author{
M. Becerra, H. Frid \\ Department of Electromagnetic Engineering, School of Engineering, Royal Institute of Technology -KTH-, \\ marley@kth.se, hfrid@kth.se \\ Stockholm, Sweden
}

\begin{abstract}
The modeling of the mechanisms of generation, loss, multiplication and transport of charge carriers is vital for the simulation of the prebreakdown process in dielectric liquids. Unfortunately, there is a lack of suitable coefficients to describe the electron generation and transport of carriers in liquids, which hinders the development of numerical models with sufficient predictive power. In this paper, the drift-dominated continuity equations for electrons and ions are coupled with Poisson's equation in order to simulate the carrier production and drift in the liquid phase under positive and negative voltages in cyclohexane. The estimations of the model are compared with measurements of current-voltage characteristics and Trichel current pulses reported in the literature for needle-plane configurations. In the analysis, the electron generation mechanisms suggested for dielectric liquids are analyzed and discussed. It is found that estimations based on the Zener equation for field-dependent molecular ionization do not agree with measurements for negative sharp points. It is also shown that the proper estimation of the electric current in the liquid phase should consider a field-dependent attachment term as well as the electrohydrodynamic movement of the liquid.
\end{abstract}

\section{INTRODUCTION}

Despite of the large amount of experimental data on the failure of dielectric liquids, no suitable model exists to explain or predict several of the observations reported in the literature [1, 2]. This has been mainly caused by the lack of knowledge on the basic processes in the liquid phase, which compared with the case of the gas and solid phases, are far less understood.

Particularly, the understanding of the mechanisms of charge carrier generation in the liquid phase and the proper modeling of the charge drift is of primary importance to formulate suitable models of breakdown. However, the conduction mechanisms at high electric fields are still not well understood in dielectric liquids [3]. Some of the mechanisms of charge generation in dielectric liquids have been described as:

a. Field emission at the cathode

b. Field ionization at the anode

c. Electric field dependent ionic dissociation

d. Electric field dependent molecular ionization

e. Electron impact ionization

The first two mechanisms are phenomena at the liquid-metal interface. Field emission is defined as the injection of electrons from a condensed phase (the cathode electrode) to another phase (the liquid) under high electric fields [4] due to tunneling. In turn, field ionization can be understood as field emission in reverse, with tunneling of electrons from the molecules of the liquid into the anode electrode [4]. These two mechanisms have been verified in cyclohexane for very small radius of curvature $\left(r_{\text {tip }}<1 \mu \mathrm{m}\right)$ [5].

The remaining processes correspond to the generation of carriers in the liquid bulk. Field dependent ionic dissociation defines the creation of ions by dissociation of neutral species (of the liquid or impurities) in the presence of an electric field, according to Onsager's theory [6]. Electric field dependent molecular ionization is a mechanism proposed by Devins and coauthors as responsible for the propagation of positive streamers [7]. This mechanism uses directly the Zener theory of tunneling in solids [8] to estimate the generation of electrons and positive ions in the liquid phase. Even though several parameters in Zener's theory are not known for liquids, recent publications have recently used this mechanism as the base for numerical models of breakdown [9-11]. However, there is little experimental evidence as to directly support the validity of this mechanism of charge generation. Last, electron collision ionization is an extension of the main mechanism of electron generation in gases. Although it is still a controversy whether electrons can gain enough energy (larger than the ionization potential) as to ionize molecules in the liquid, Haidara and Denat [12] has estimated the multiplication coefficient $\alpha$ for cyclohexane.

In order to contribute to the discussion about the mechanisms of generation of carriers in liquids, this paper intends to use only the electron generation mechanisms in a numerical model and to compare the obtained results directly with experimental data. For this, the well-documented voltagecurrent characteristics measured of Denat and coauthors [5] in cyclohexane are taken as the experimental base to compare with the predictions of the electron generation mechanisms. Only field emission, electric field dependent molecular ionization and electron impact ionization are considered.

\section{NUMERICAL MODEL}

The continuity equations describing the growth of the density of charged carriers (electrons $\rho_{e}$, positive ions $\rho_{p}$ and negative ions $\rho_{n}$ ) in a liquid (as in a gas [13]) can be written as [9]:

$$
\begin{gathered}
\frac{\partial \rho_{e}}{\partial t}+\nabla \cdot \rho_{e} \mu_{e} \vec{E}=G(|\vec{E}|)-A-\frac{R_{p e} \rho_{p} \rho_{e}}{q} \\
\frac{\partial \rho_{p}}{\partial t}-\nabla \cdot \rho_{p} \mu_{p} \vec{E}=G(|\vec{E}|)-\frac{R_{p e} \rho_{p} \rho_{e}}{q}-\frac{R_{p n} \rho_{p} \rho_{n}}{q} \\
\frac{\partial \rho_{n}}{\partial t}+\nabla \cdot \rho_{n} \mu_{n} \vec{E}=A-\frac{R_{p n} \rho_{p} \rho_{n}}{q}
\end{gathered}
$$


where $\mu_{e}, \mu_{p}$ and $\mu_{n}$ are the mobilities for electrons and ions, and $R_{p e}, R_{p n}$ are the electron-ion and ion-ion recombination rates. The electron generation term in the liquid bulk $G$ is function of the electric field. For electron-dependent molecular ionization, the source term $G$ is expressed as:

$$
G^{F I}(|\vec{E}|)=\frac{q^{2} n_{0} a|\vec{E}|}{h} \exp \left(-\frac{\pi^{2} m^{*} a \Delta^{2}}{q h^{2}|\vec{E}|}\right)
$$

where $h$ is Plank's constant, $a$ is the molecular separation distance, $m^{*}$ is the effective electron mass, $n_{0}$ is the number density of ionizable species and $\Delta$ is the liquid ionization potential [7]. For electron impact ionization, the source term $G$ is expressed as:

$$
G^{C I}(|\vec{E}|)=\alpha \rho_{e} \mu_{e}|\vec{E}|=\left(A_{G} \exp \left(-\frac{B_{G}}{|\vec{E}|}\right)\right) \rho_{e} \mu_{e}|\vec{E}|
$$

where $A_{G}$ and $B_{G}$ are fitting constants to the multiplication coefficient estimated in [12].

The boundary condition for the electron density injected by field emission at the electrode is calculated with the FowlerNordheim equation:

$$
\begin{gathered}
\rho_{e}^{\text {(cathode) }}=\frac{\frac{a_{F N}|\vec{E}|}{\phi} \exp \left(\frac{-v\left(f_{F N}\right) b_{F N} \phi^{1.5}}{|\vec{E}|}\right)}{\mu_{e}} \\
v\left(f_{F N}\right)=1-f_{F N}+0.166 f_{F N} \ln f_{F N} \\
f_{F N}=\frac{1.4299 \mathrm{eV}^{2} \mathrm{~V}^{-1} \mathrm{~nm}|\vec{E}|}{\phi^{2}}
\end{gathered}
$$

where $\phi$ is the metal work function and the parameters $a_{F N} \approx 1.5414 \cdot 10^{-6} \mathrm{AeVV}^{-2}$ and $b_{F N} \approx 6.8308 \mathrm{eV}^{-1.5} \mathrm{Vnm}^{-1}[14]$.

The attachment loss term $A$ is usually defined in the literature [9-11] through the attachment rate $\tau_{a}$ as:

$$
A=\frac{\rho_{e}}{\tau_{a}}
$$

\section{SIMULATION OF CURRENT-VOLTAGE CHARACTERISTICS FOR SHARP POINTS}

\section{A. Field emission at the cathode}

The current-voltage (IV) characteristics for sharp cathode points (with tip radius smaller than $1 \mu \mathrm{m}$ ) reported by Denat and coworkers have been described in terms of field emission at the cathode [5]. By using Fowler-Nordheim plots and the Harper-Gomer analysis of the space charge limited regime of their measurements, average estimations of the tip radius and the negative ion mobility were obtained [5]. In order to perform a more detailed analysis of the field emission at the cathode in that experiment, the model described in the previous section is used. As in the literature [9-11], the negative ion mobility $\mu_{n}$ is taken as the average electrohydrodynamic (EHD) mobility $\left(10^{-7} \mathrm{~m}^{2} \mathrm{~V}^{-1} \mathrm{~s}^{-1}\right.$ [5]) while the attachment rate constant $\tau_{a}$ is taken from the measured zero-field lifetime of electronic charges (500 ns for cyclohexane [5]). As shown in Fig. 3, there is an acceptable agreement between measurements and the IV characteristic simulated using these values. However, it is incorrectly estimated that a large amount of electrons drift all the way to the anode (Fig. 2). Since the effective attachment rate in liquids is a function of electric field [15], a constant, zero-field attachment rate does not reproduce the fact that attachment is dominant at low fields [12]. This leads to the overestimation of the electron density in areas away, and the underestimation of the negative ion density in the region close to the cathode. In turn, if the attachment term $A$ in (1) is defined as a function of electric field as in gas discharge simulations [13], a slightly worse agreement is found between the calculations and the measurement (Fig. 1). However, a more realistic estimation of the electron density distribution is reached in this case.

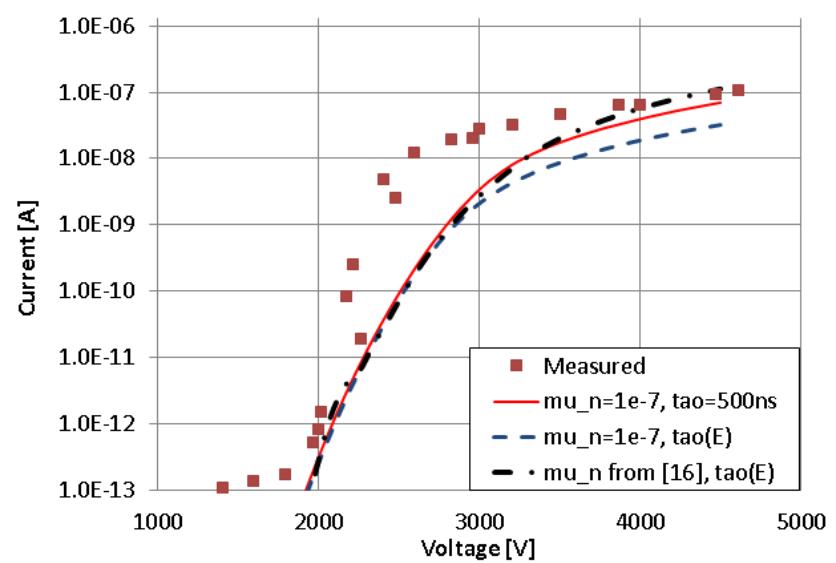

Fig. 1. Comparison of the IV characteristic for sharp negative points measured in [5] and simulated with only field emission for different values of the negative ion mobility $\mu_{n}$ and the attachment rate constant $\tau_{a}$.

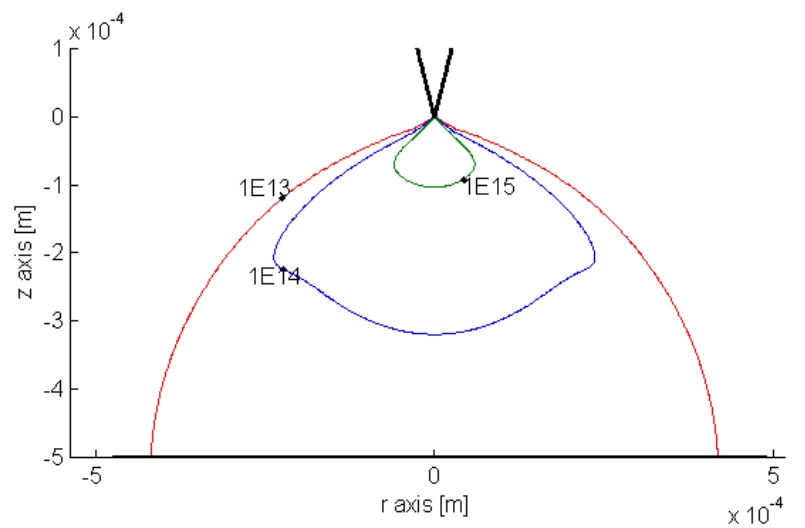

Fig. 2. Estimated number density of electrons for an applied voltage of $-3000 \mathrm{~V}$. The calculations are performed by assuming $\mu_{n}=10^{-7} \mathrm{~m}^{2} \mathrm{~V}^{-1} \mathrm{~s}^{-1}$ and $\tau_{a}=500 \mathrm{~ns}$.

A better agreement between the measurements and the estimations with the field-dependent attachment term along the space charge limited regime can be reached with a proper representation of the negative ion movement. Note that the assumption of the average EHD mobility for negative ions does not reflect the actual mobility of negative ions due to the 
liquid movement. Calculations considering the electrostatic force acting on the space charge transported by the moving liquid have shown that the actual negative ion mobility in the proximity of the cathode point is larger than the average EHD mobility [16]. This would lead to a better agreement between the calculations and the measurements in the space charge limited regime where EHD liquid motion becomes significant (Fig. 1).

\section{B. Electric field dependent molecular ionization}

The major challenge of using equation (4) to describe the generation of electrons in the liquid bulk is the selection of several of its parameters for dielectric liquids [9]. Particularly, the selection of the molecular separation distance $a$ and the number density of ionizable species $n_{0}$ is difficult since they are not usually known.

However, in order to estimate the IV characteristic of positive sharp points, let us assume that field dependent molecular ionization is the main carrier generation mechanism. For that, the parameters $a, m^{*}$ and $n_{0}$ are first assumed equal to the corresponding values used in [9] for naphthenic molecules (such as cyclohexane). As it can be seen in Fig. 3, the estimations using these parameters strongly disagree with the IV characteristic measured in [5].

Better values of $a$ and $n_{0}$ are estimated by fitting the simulated IV characteristic to the measured values. Interestingly, it is found that estimations using (4) can reproduce the complete IV characteristics of positive sharp points very well when the right "fitting" parameters are used (Fig. 3).

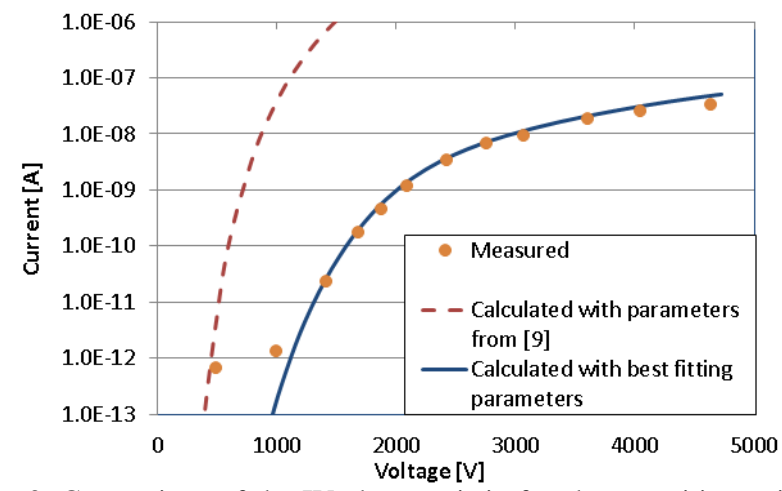

Fig. 3. Comparison of the IV characteristic for sharp positive points measured in [5] and simulated with only field-dependent molecular ionization mechanism for different parameters.

In order to crosscheck whether field-dependent molecular ionization takes place indeed in the liquid bulk, equation (4) is also used (in addition to field emission at the electrode) to estimate the IV characteristic in negative sharp points. At first, the best fitting parameters from the simulation presented in Fig. 3 are used. Unexpectedly, it is found that (4) estimate currents several orders of magnitude larger than those measured in the negative point configuration at low voltages (Fig. 4). This is caused by the overestimation on the electron generation in the liquid bulk at relatively low electric fields when (4) is used. Thus, it can be concluded that even though Zener's equation (4) can be fitted to reach agreement with the IV characteristic in positive points, it fails to predict the currents measured for negative points. This result cast serious doubts on the validity of the field-dependent molecular ionization as a mechanism of electron generation in cyclohexane. Moreover, it can be noted that the estimations are even worse when the parameters suggested in [9] are used. Hence, it is also doubtful that field-dependent molecular ionization following [9] can be used to model negative streamers in hydrocarbon liquids as recently suggested [17].

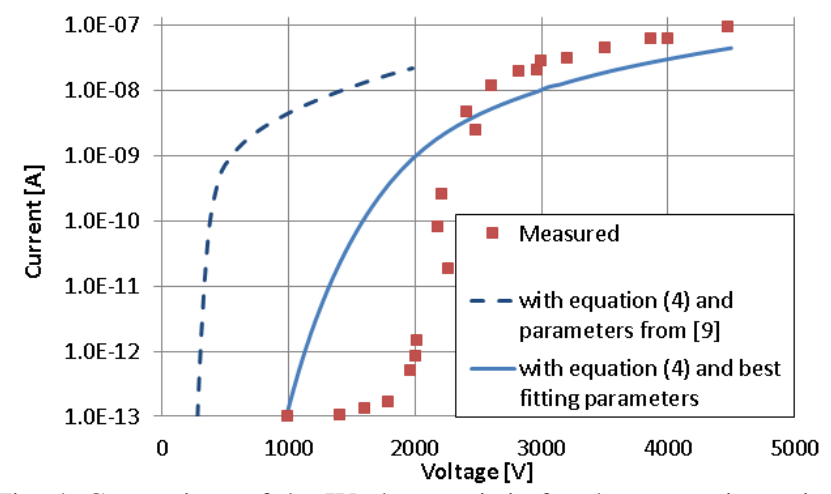

Fig. 4. Comparison of the IV characteristic for sharp negative points measured in [5] and simulated with field emission at the cathode and field-dependent molecular ionization mechanism.

\section{Electron impact ionization}

Since the multiplication coefficient $\alpha$ has been explicitly expressed as a function of electric field [12], electron impact ionization can be easily implemented in the model. In the case of sharp negative points, the inclusion of impact ionization leads to a better agreement of the calculations with the measurements (Fig. 5). This indicates that impact ionization has a secondary, but still significant role on the electron generation of negative points in cyclohexane. In the case of sharp positive points, the multiplication of electrons by impact ionization is very limited even if enough seed free electrons are released at the edge of the area where $\alpha$ ionization is larger than attachment $\eta(\alpha-\eta>0)$.

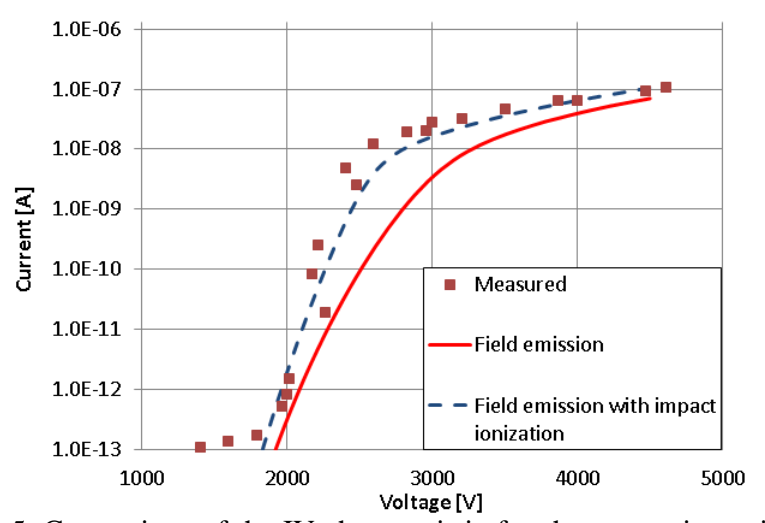

Fig. 5. Comparison of the IV characteristic for sharp negative points measured in [5] and simulated with field emission at the cathode and impact ionization based on [12]. 


\section{SIMULATION OF TIME-VARYING CURRENTS FOR LARGER RADIUS NEGATIVE POINTS}

An additional analysis of the electron generation mechanism can be performed for larger negative points with radius larger than $1 \mu \mathrm{m}$. For this case, it is relevant to assess whether the model can reproduce the Trichel pulses measured when a voltage of $2500 \mathrm{~V}$ is applied to a $1 \mu \mathrm{m}$ needle point [5]. Considering the discussion in Section II.B, the simulations consider field emission and impact ionization only.

As it can be seen in Fig. 6, the simulated current in the configuration once a single seed free electron appears at the point tip is several orders of magnitude smaller than the measured values (with a peak of about $100 \mu \mathrm{A}$ [5]). This result shows that an additional mechanism of electron transfer into the liquid (other than the ones described above) is required in the simulation in order to reach the measured currents. For this reason, it is relevant to consider that the point electrode may not be perfectly rounded or oxide-free. In such a case, the effective electric field at the point surface could deviate from the values calculated for a perfectly smooth tip or there could be accumulation of positive ions which are not readily neutralized and form a space charge due to a semi-insulating layer on the electrode [18].

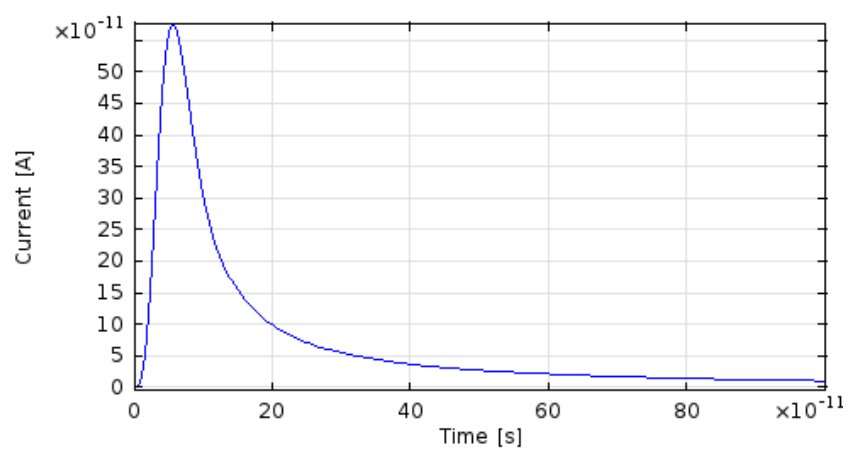

Fig. 6. Simulated current for a $1 \mu \mathrm{m}$ negative point under $2500 \mathrm{~V}$ considering field emission and impact ionization.

\section{CONCLUSIONS}

In this paper, a numerical model is used to simulate the IV characteristics for negative and positive voltages in the needleplane geometry. It is shown that a field-dependent attachment rate is required in order to properly estimate the electron density in low field areas for negative points. In the same manner, it is shown that better estimations of the IV characteristic are reached when the effective (instead of a constant average) EHD mobility is assumed for negative ions. It is also found that even though Zener's equation for fielddependent molecular ionization mechanism can be easily fitted to reach excellent agreement with the IV curve for positive points, it fails to reproduce measured results for negative points. This result cast serious doubts on the validity of the field-dependent molecular ionization as a mechanism of electron generation in hydrocarbon liquids such as cyclohexane. On the other hand, it is found that simulations including field emission at the cathode and electron impact ionization alone do not reproduce the Trichel current measured for negative points with larger radius. Thus, it is clear that another mechanism of electron generation is necessary in order to explain the large Trichel pulses measured in the laboratory.

\section{ACKNOWLEDGMENT}

M.B. would like to acknowledge the financial support of the Swedish strategic research program StandUp for Energy.

\section{REFERENCES}

[1] O. Hestad, L. Lundgaard, D. Linhjell, "New experimental system for the study of the effects of tempearture and liquid to solid transition on streamers in dielectric liquids: application to cyclohexane”, IEEE Trans. Diel. Electr. Insul., Vol 17, No. 3, pp. 764-774, 2010

[2] Beroual, A.; Zahn, M.; Badent, A.; Kist, K.; Schwabe, A. J.; Yamashita, H.; Yamazawa, K.; Danikas, M.; Chadband, W.G.; Torshin, Y., "Propagation and structure of streamers in liquid dielectrics," Electrical Insulation Magazine, IEEE , vol.14, no.2, pp.6,17, March-April 1998

[3] R. E. Tobazeon, "Streames in liquids", in: the Liquid state and its electrical properties, E. E., Kundhart, NATO series, 193, 1987.

[4] R. Gomer, Field emission and field ionization, American Institute of Physics, Harvard University Press, NY, 1993.

[5] Denat, A.; Gosse, J-P; Gosse, B., "Electrical conduction of purified cyclohexane in a divergent electric field", IEEE Transactions on Electrical Insulation, vol.23, no.4, pp.545,554, Aug 1988.

[6] Onsager, L., "Deviations from Ohm's law in weak electrolytes”, J. Chem. Phys, 2, 1934, pp. 599

[7] Devins, J. d., Rzad, S.J., Schwabe, R. J., "Breakdown and prebreakdown phenomena in liquids”, J. Appl, Phys., 52, 1981, pp. 4521

[8] Zener, C., A, "Theory of the Electrical Breakdown of Solid Dielectrics", Proc. R. Soc. Lond. A 2 July 1934 vol. 145 no. 855 523-529.

[9] J. Hwang, M. Zahn, L. Pettersson, "Mechanisms behind positive streamers and their distinct propagation modes in transformer oil", IEEE Transactions on Dielectrics and Electrical Insulation, vol.19, no.1, pp.162,174, February 2012, doi: 10.1109/TDEI.2012.6148515

[10] Jiang, C, Lewin, P L and Sima, W (2012) Modelling the Streamer Process in Liquid Dielectrics. In, The Fifth UHVnet Colloquium, University of Leicester, Leicester, UK, 18 - 19 Jan 2012. , 33.

[11] Joshi, R.P., Qian, J., Zhao, G., Kolb, J., Schoenbach, K. H., Schamiloglu, E., Gaudet, J., Are microbubbles necessary for the breakdown of liquid water subjected to a submicrosecond pulse? J. Appl. Phys. 96, 5129 (2004).

[12] Haidara, M.; Denat, A., "Electron multiplication in liquid cyclohexane and propane: an estimation of the ionization coefficient," Conduction and Breakdown in Dielectric Liquids, 1990. ICDL 1990. 10th International Conference on , vol., no., pp.397,401, 10-14 Sep 1990

[13] Davies, A., Davies, C., Evans, C., Computer simulation of rapidly developing gaseous discharges, Proc. IEE, Vol 118, No.6, 1971, pp. 816-824.

[14] Forbes, Richard G.; Deane, Jonathan H.B. (2007). "Reformulation of the standard theory of Fowler-Nordheim tunnelling and cold field electron emission". Proceedings of the Royal Society A: Mathematical, Physical and Engineering Sciences 463 (2087): 2907.

[15] W. Schmidt, Hot electron mobility and electron attachment in non-polar fluids, in The Liquid State and Its Electrical Properties, Edited by E. Kundhardt, L. Christophoou and L. Luessen, Series B: Physics Vol. 193, New York, Plenum Press, pp. 273-282

[16] H. Frid, M. Becerra, Simulation of Microbubbles during the Initial Stages of Breakdown in Cyclohexane, Annual Report IEEE Conference on Electrical Insulation and Dielectric Phenomena, 2013

[17] J. Jadidian, M. Zahn, N. Lavesson, O. Widlund, K. Borg, "Surface Flashover Breakdown Mechanism on the Liquid Immersed Dielectrics," Applied Physics Letters, Vol. 100, p. 172903, April 2012.

[18] J. Lewis, An overview of electrical processes leading to dielectric breakdown in liquids, in: the Liquid state and its electrical properties, E. E., Kundhart, NATO series, 193, 1987, pp. 431-453. 